DFTT $43 / 95$

August 1995

\title{
Calogero-Sutherland techniques in the physics of disordered wires
}

\author{
M. Caselle [ \\ Istituto Nazionale di Fisica Nucleare, Sezione di Torino \\ Dipartimento di Fisica Teorica dell'Università di Torino \\ via P.Giuria 1, I-10125 Turin,Italy
}

\begin{abstract}
We discuss the connection between the random matrix approach to disordered wires and the Calogero-Sutherland models. We show that different choices of random matrix ensembles correspond to different classes of CS models. In particular, the standard transfer matrix ensembles correspond to CS model with sinh-type interaction, constructed according to the $C_{n}$ root lattice pattern. By exploiting this relation, and by using some known properties of the zonal spherical functions on symmetric spaces we can obtain several properties of the Dorokhov-Mello-Pereyra-Kumar equation, which describes the evolution of an ensemble of quasi one-dimensional disordered wires of increasing length $L$. These results are in complete agreement with all known properties of disordered wires.
\end{abstract}

${ }^{1}$ caselle@to.infn.it 


\section{Introduction}

During last years an increasing interest has been attracted by the physics of quantum electronic transport in disordered wires [回]. One of the main reasons for this interest lies in the high degree of universality of some experimental observations. In particular it was shown that the variations of the measured conductance as a function of the magnetic field or of the Fermi energy are independent of the size and degree of disorder of the sample and have a variance always of order $e^{2} / h$. This phenomenon is usually known as universal conductance fluctuations (UCF). Its universality suggests that disordered wires could be described by some relatively simple Hamiltonian, independent of the particular model or disorder realization. This approach was pioneered by Imry [2] and developed by Muttalib, Pichard and Stone [3], who suggested to describe UCF by constructing a Random Matrix Theory (RMT) of quantum transport, in analogy to the Wigner-Dyson RMT for nuclear energy

levels. However it was soon realized that, besides the obvious analogies, there are some relevant differences between the Wigner-Dyson ensembles (WDE) of random matrices and those constructed to describe the physics of disordered wires (which we shall denote in the following as Transfer Matrix ensembles (TME)). The most interesting feature of these TME is the presence of a Fokker-Plank type evolution equation, known as DMPK equation (see below) which can be considered the equivalent in the context of TME of the Brownian motion approach to WDE suggested by Dyson [4] (for a discussion of these analogies see [5]).

The aim of this contribution is to show that the relationship between WDE and TME (and the parallel one between Brownian motion and DMPK equation) can be well understood by exploiting their equivalence with the so called "CalogeroSutherland" (CS) models [6] which are quantum integrable models describing a set of $N$ particles moving along a line (see below). In particular it will be shown that the Schrödinger equation of the CS models is equivalent to the DMPK equation and that WDE's, TME's and the S-matrix ensembles described in [5] correspond to different realizations of the CS models. We shall show that the common, underlying, mathematical structure of all these models is the theory of Laplace-Beltrami operators on Symmetric Spaces. By using some recent results on the eigenfunctions of these operators, which are known as "zonal spherical functions" we shall construct exact (for the unitary ensemble) or asymptotic (in the other cases) solutions of the DMPK equation and describe several physical properties of disordered wires.

This contribution is organized as follows: after a short introduction to disorder wires and to their TME description (sect.2) we shall discuss the DMPK equation (sect.3). In Sect. 4 we shall give a short introduction to the CS models and show the anticipated equivalence with the TME. Sect. 5 will be devoted to the solution of the DMPK equation and sect.6 to some concluding remarks. 


\section{Disordered wires}

The peculiar feature of disordered wires is that in these systems, at low enough temperature, the phase coherence of the electron's wavefunctions is kept over distances much larger than the typical mean free path, thus allowing to study several non-trivial quantum effects. These phenomenon can be studied only at low temperatures where the inelastic electron-phonon scattering (which changes the phases of electrons in a random way) becomes negligible and resistivity is completely dominated by the scattering against random impurities, which is elastic and changes the phases in a reproducible, deterministic way. The natural theoretical framework to describe these systems is the Landauer theory [7] which assumes the electrons in thermal equilibrium with the various chemical potentials in the leads. The disordered wire, with its impurities, is then regarded as a scattering center for the electrons originating from the current leads and the conductance $G$ is proportional to the transmission coefficients of the scattering problem. Within this approach Fisher and Lee [8] proposed the following expression for the conductance in a twoprobe geometry (namely a finite disordered section of length $L$ and transverse width $W$, to which current is supplied by two semi-infinite ordered leads):

$$
G=G_{0} \operatorname{Tr}\left(t t^{\dagger}\right) \equiv G_{0} \sum_{n} T_{n}, \quad G_{0}=\frac{2 e^{2}}{h} .
$$

where $t$ is the $N \times N$ transmission matrix of the conductor and $T_{1}, T_{2} \cdots T_{N}$ are the eigenvalues of the product $t t^{\dagger}$ and are usually called transmission eigenvalues. $N$ is the number of scattering channels at the Fermi level. $N$ depends on the width of the wire and even in the narrowest metal wires it is of the order of $N \sim 10^{4}-10^{5}$ so that for metal wires a large $N$ approximation will give in general very good results (notice however that semiconductor microstructure with very low values of $N$ can be constructed and studied). In the following we shall often refer to the dimensionless conductance $g$, defined as $g \equiv G / G_{0}$. The transmission matrix $t$ is a component of the $2 N \times 2 N$ scattering matrix $S$ which relates the incoming flux to the outgoing flux:

$$
\begin{gathered}
S\left(\begin{array}{c}
I \\
I^{\prime}
\end{array}\right)=\left(\begin{array}{c}
O \\
O^{\prime}
\end{array}\right), \\
S=\left(\begin{array}{cc}
r & t \\
t^{\prime} & r^{\prime}
\end{array}\right),
\end{gathered}
$$

where I,O,I',O' are $N$ component vectors which describe the incoming and outgoing wave amplitudes on the left and right respectively, and $r$ is the $N \times N$ reflection matrix. Current conservation implies

$$
|I|^{2}+\left|I^{\prime}\right|^{2}=|O|^{2}+|O|^{2}
$$

which is equivalent to the requirement of unitarity: $S \in U(2 N)$. However it turns out that for the problem that we are studying a much better parametrization is 
given by the transfer matrix $M$ which is defined as:

$$
M\left(\begin{array}{c}
I \\
O
\end{array}\right)=\left(\begin{array}{c}
O^{\prime} \\
I^{\prime}
\end{array}\right),
$$

The relation between the transfer matrix and the transmission eigenvalues becomes clear if one constructs an auxiliary matrix $Q$ defined in terms of $M$ as follows:

$$
Q=\frac{1}{4}\left[M^{\dagger} M+\left(M^{\dagger} M\right)^{-1}-2\right]
$$

The eigenvalues $\left\{\lambda_{i}\right\}$ of $Q$ are non-negative and can be related to the transmission eigenvalues $T_{i}$ by

$$
\lambda_{i} \equiv\left(1-T_{i}\right) / T_{i}
$$

The physics of the disordered wires that we are studying will be completely described if we can obtain the probability distribution $P\left(\left\{\lambda_{i}\right\}\right)$ for the eigenvalues $\lambda_{i}$ (and consequently for the $T_{i}$ 's).

To this end let us first study some general properties of the transfer matrix which are direct consequences of the physical symmetries of the problem. First of all, it is easy to see that the same flux conservation constraint eq.(价 discussed above implies in this case the conservation of a hyperbolic norm:

$$
M^{\dagger} \Sigma_{z} M=\Sigma_{z}
$$

with

$$
\Sigma_{z}=\left(\begin{array}{cc}
1 & 0 \\
0 & -1
\end{array}\right),
$$

where $\mathbf{0}$ and $\mathbf{1}$ are the zero and unit $N \times N$ matrices. As a consequence of (8), $M \in S U(N, N)$. The ensemble of transfer matrices defined in this way is usually called "unitary ensemble" (ensemble IIa in the notation of ref. [9]).

If the system is also invariant under time reversal symmetry, $M$ must satisfy a further constraint:

$$
M^{*} \Sigma_{x} M=\Sigma_{x}
$$

with

$$
\Sigma_{x}=\left(\begin{array}{ll}
\mathbf{0} & \mathbf{1} \\
\mathbf{1} & \mathbf{0}
\end{array}\right),
$$

It is possible to show that the joint application of (8) and (10) implies $M \in$ $S P(2 N, \mathbf{R})$ 10]. The ensemble of transfer matrices defined in this way is usually called "orthogonal ensemble" (ensemble I in the notation of ref. [9]).

From an experimental point of view it is very simple to control the time reversal symmetry which is eliminated by the application of an external magnetic field.

If the disordered wire contains "magnetic impurities", namely if the spin-orbit interaction in the scattering against impurities becomes important then the spinrotation symmetry (which was implicitly assumed in all the above discussion) is not 
any more conserved. In this case the two spin components of the electrons must be treated separately. Each one of the input and output vectors $I, I^{\prime}, O, O^{\prime}$ becomes a collection of $N$ spinors (one for each channel) and each spinor contains the two spin degrees of freedom. Hence $M$ is in this case a $4 N \times 4 N$ complex matrix. If only flux conservation is imposed (time reversal symmetry broken) then $M \in U(2 N, 2 N)$ and we find again the unitary ensemble described above with the only change: $N \rightarrow 2 N$ (this difference was kept explicit in ref. [9], where this case was denoted as IIb to distinguish it from the spin-rotation symmetric unitary ensemble IIa). If time reversal symmetry is conserved (namely if there are magnetic impurities, but no external magnetic field) then one must impose the following constraint on $M$ [1] (analogous of that of eq.(100)

$$
M^{*}=K M K^{T}
$$

with

$$
K=\left(\begin{array}{cc}
\mathbf{0} & \mathbf{A} \\
\mathbf{A} & \mathbf{0}
\end{array}\right)
$$

where $\mathbf{A}$ is a $2 N \times 2 N$ block diagonal matrix

$$
\mathbf{A}=\sigma \mathbf{1}, \quad \sigma=\left(\begin{array}{cc}
0 & 1 \\
-1 & 0
\end{array}\right)
$$

This constraint implies that $M \in S O^{*}(4 N)$ and defines the so called symplectic ensemble (ensemble III in the language of ref [9]).

The second step in order to construct the probability distribution for the $\lambda_{i}$ 's comes from the identification of the $\lambda_{i}$ 's themselves as the relevant physical degrees of freedom of the system. This identification has some very interesting group theoretical consequences. In fact the choice of the $\lambda_{i}$ 's as relevant physical parameters induces the following parametrization for $M$ for the orthogonal and unitary cases [10]:

$$
\begin{aligned}
M & =\left(\begin{array}{cc}
u^{(1)} & \mathbf{0} \\
\mathbf{0} & u^{(2)}
\end{array}\right)\left(\begin{array}{cc}
\sqrt{1+\Lambda} & \sqrt{\boldsymbol{\Lambda}} \\
\sqrt{\boldsymbol{\Lambda}} & \sqrt{1+\boldsymbol{\Lambda}}
\end{array}\right) \\
& \times\left(\begin{array}{cc}
u^{(3)} & \mathbf{0} \\
\mathbf{0} & u^{(4)}
\end{array}\right) \equiv U \Gamma V
\end{aligned}
$$

where $\boldsymbol{\Lambda}$ is a $N \times N$ real, diagonal, matrix with entries the eigenvalues $\lambda_{1}, \lambda_{2}, \cdots \lambda_{N}$ in both cases. The $u^{(i)},(i=1,2,3,4)$ are 4 independent $N \times N$ unitary matrices in the unitary case while in the orthogonal case they are constrained by the relations:

$$
u^{(2)}=u^{(1) *}, u^{(4)}=u^{(3) *}
$$

In the symplectic case we have again the same parametrization, if the various matrices are written in terms of quaternions. Thus in this case $\boldsymbol{\Lambda}$ is a $N \times N$ 
quaternion real, diagonal matrix and the $u^{(i)}$ 's are $N \times N$ quaternion, unitary matrices which again obey the constraint (16).

In this parametrization we recognize a $G / H$ coset structure, where $G$ is the group to which the transfer matrix belongs and $H$ is the group to which the $U$ and $V$ matrices belong. The coset structure is immediately evident if we notice that any transformation $M \rightarrow M^{\prime}=W M W^{-1}$, with $W \in H$ gives again a transfer matrix which can be decomposed as $M^{\prime}=U^{\prime} \Gamma V^{\prime}$ with $U^{\prime}=W U, V^{\prime}=V W^{-1}$ and the same matrix $\Gamma$. So the physically relevant parameters $\lambda_{i}$ are left unchanged by such transformation and thus belong to the coset space $G / H$. These cosets are listed in Tab.1 for the three ensembles in which we are interested.

\begin{tabular}{|c|c|c|c|}
\hline$G$ & $H$ & $\mathrm{~T}$ & $\mathrm{~S}-\mathrm{R}$ \\
\hline$S p(2 N, \mathbf{R})$ & $U(N)$ & $y$ & $y$ \\
$S U(N, N)$ & $S U(N) \otimes S U(N) \otimes U(1)$ & $n$ & $y$ \\
$S O^{*}(4 N)$ & $U(2 N)$ & $y$ & $n$ \\
\hline
\end{tabular}

Table 1: $G / H$ cosets for the orthogonal (first line), unitary (second line) and symplectic (last line) ensembles. In the first two columns the group $G$ and the subgroup $H$. In the last two columns the status of the time reversal $(\mathrm{T})$ and spin rotation $(\mathrm{S}-\mathrm{R})$ symmetries respectively $(\mathrm{y}=$ conserved, $\mathrm{n}=$ broken$)$.

However this is not the end of the story. Looking at the three particular realizations of the pair $G, H$ listed in tab. 1 we see that in all the three cases the cosets are actually symmetric spaces (see tab.2). What is more important, we recognize in the parametrization (15) the so called "spherical decomposition" of those symmetric spaces (see for instance [12]). This tells us that the $\lambda_{i}$ 's play the role of (generalized) radial coordinates in $G / H$ and implies that the $\lambda_{i}$ 's are also invariant under (generalized) angular transformations in $G / H$ and that only the radial projection of any given dynamical operator will influence their dynamics.

At this point the only remaining step is to impose some dynamical principle so as to obtain an "equation of motion" for the probability distribution of the $\lambda_{i}$ 's.

\section{The DMPK equation.}

This program was completed during the eighties, at least in the case of quasi onedimensional wires, by Dorokhov [13], and independently by Mello, Pereyra, and Kumar [10] (for $\beta=1$ ) by looking at the infinitesimal transfer matrix describing the addition of a thin slice to the wire. The resulting evolution equation for the eigenvalue distribution $P\left(\left\{\lambda_{i}\right\}, s\right)$ is usually known as Dorokhov-Mello-PereyraKumar (DMPK) equation. The only assumptions which are needed to obtain this equation are first that the conductor must be weakly disordered so that the scattering in the thin slice can be treated by using perturbation theory and second 
that the flux incident in one scattering channel is, on average, equally distributed among all outgoing channels. It is exactly this second assumption which restricts the DMPK equation to the quasi 1-d regime, where finite time scale for transverse diffusion can be neglected. The results of [10] were then generalized to $\beta=2,4$ in Refs. [14, 11]. The DMPK equation is:

$$
\frac{\partial P}{\partial s}=D P
$$

where $s$ is the length $L$ measured in units of the mean free path $l: s \equiv L / l$ and $D$ can be written in terms of the $\lambda_{i}$ 's as follows:

$$
D=\frac{2}{\gamma} \sum_{i=1}^{N} \frac{\partial}{\partial \lambda_{i}} \lambda_{i}\left(1+\lambda_{i}\right) J(\lambda) \frac{\partial}{\partial \lambda_{i}} J(\lambda)^{-1},
$$

with $\gamma=\beta N+2-\beta . \beta \in\{1,2,4\}$ is the symmetry index of the ensemble of scattering matrices: $\beta=1$ for the orthogonal ensemble, $\beta=2$ for the unitary ensemble and $\beta=4$ for the symplectic one, in full analogy with the well know Wigner-Dyson classification. $J\left(\left\{\lambda_{n}\right\}\right)$ denotes the Jacobian from the matrix to the eigenvalue space:

$$
J\left(\left\{\lambda_{n}\right\}\right)=\prod_{i<j}\left|\lambda_{j}-\lambda_{i}\right|^{\beta}
$$

There is however a completely independent, and very elegant, way to obtain the DMPK equation. Let us assume as dynamical principle to obtain an equation of motion for $P(\lambda)$ the simplest possible choice compatible with the constraints described in sect.2. That is, let us assume that, as $L$ (the length of the wire) increases, the matrix $M$ freely diffuses in the $G / H$ space going from the perfectly conducting limit $(L=0)$ (which plays the role of initial condition for this evolution equation) to the insulating, localized limit $(L=\infty)$. In general a free diffusion in $G / H$ is described by the Laplace-Beltrami operator of $G / H$. However, since the $\lambda$ 's are the radial coordinates of $G / H$, their behaviour as a function of $L$ will only depend on the radial part $B$ of the Laplace-Beltrami operator. The resulting equation is:

$$
\frac{\partial P}{\partial s}=\alpha B P
$$

where $\alpha$ is a (for the moment undetermined) diffusion constant and $B$ is defined as follows:

$$
B=[\xi(x)]^{-2} \sum_{k=1}^{n} \frac{\partial}{\partial x_{k}}[\xi(x)]^{2} \frac{\partial}{\partial x_{k}},
$$

where we have chosen the following parametrization for the radial coordinates of the manifold: $\lambda_{i}=\sinh ^{2} x_{i}$, and

$$
\xi(x)=\prod_{i<j}\left|\sinh ^{2} x_{j}-\sinh ^{2} x_{i}\right|^{\frac{\beta}{2}} \prod_{i}\left|\sinh 2 x_{i}\right|^{\frac{1}{2}} .
$$


It is now only matter of straightforward algebra to recognize that $B$ and the DMPK operator $D$ are related by:

$$
D=\frac{1}{2 \gamma}[\xi(x)]^{2} B[\xi(x)]^{-2},
$$

thus allowing, through a suitable choice of $\alpha$ and normalization of $P(\lambda)$ to identify eq.(17) and eq.(20). This identification was first recognized by Hüffmann [15, and has been recently discussed in [16] and [17].

Eq.(20) can be considered the analogous, in the context of the TME's (hence for symmetric spaces of negative curvature) of the Brownian motion approach in the case of S-matrix ensembles (which as we shall show below are characterized, by the same symmetric spaces, but with positive curvature).

An important and unexpected property of the DMPK equation is that if $\beta=2$ the various $\lambda_{i}$ can be decoupled. This was recently realized by Beenakker and Rejaei [18] who showed that the DMPK equation can be rewritten as Schrödingerlike equation (in imaginary time) for a set of $N$ interacting fermions. The mapping was obtained by setting: $\lambda_{n}=\sinh ^{2} x_{n}$, and by making the following substitution

$$
P\left(\left\{x_{n}\right\}, s\right)=\xi(x) \Psi\left(\left\{x_{n}\right\}, s\right) .
$$

In this way the DMPK equation becomes exactly equivalent to:

$$
\begin{gathered}
-\frac{\partial \Psi}{\partial s}=(\mathcal{H}-U) \Psi \\
\mathcal{H}=-\frac{1}{2 \gamma} \sum_{i}\left(\frac{\partial^{2}}{\partial x_{i}^{2}}+\frac{1}{\sinh ^{2} 2 x_{i}}\right) \\
+\frac{\beta(\beta-2)}{2 \gamma} \sum_{i<j} \frac{\sinh ^{2} 2 x_{j}+\sinh ^{2} 2 x_{i}}{\left(\cosh 2 x_{j}-\cosh 2 x_{i}\right)^{2}} \\
U=-\frac{N}{2 \gamma}-N(N-1) \frac{\beta}{\gamma}-N(N-1)(N-2) \frac{\beta^{2}}{6 \gamma} .
\end{gathered}
$$

By choosing $\beta=2$, the remaining interaction terms among the $x_{n}$ disappear, the equation can be decoupled and can be solved exactly [18.

This equivalence with a Schrödinger equation is another feature of the DMPK equation which has a natural explanation in the framework of the Calogero-Sutherland models.

\section{Calogero-Sutherland Models}

These models describe $N$ particles on a line, identified by their coordinates $\left\{x_{i}\right\}, i=$ $1 \cdots N$, interacting (at least in the simplest version of the models) with a pairwise potential $f\left(x_{i}, x_{j}\right)$. Several realizations of this potential have been studied in the 
literature (for a comprehensive review see ref. [19]), but in the following we shall mainly be interested in only two realizations. The sin-type CS model, in which $f\left(x_{i}, x_{j}\right)=1 / \sin ^{2}\left(x_{i}-x_{j}\right)$ and the sinh-type for which $f\left(x_{i}, x_{j}\right)=1 / \sinh ^{2}\left(x_{i}-x_{j}\right)$.

The most relevant feature of these models is that (under particular conditions discussed below, see eq.(28) and (34)) they have $N$ commuting integrals of motions, they are completely integrable and their Hamiltonian can be mapped into the radial part of a Laplace-Beltrami operator on a suitable symmetric space. In particular we have spaces with negative curvature for the sinh models and of positive curvature for the sin-type ones.

In the original formulation of the CS model, the interaction among the particles was simply pairwise [6]. But it was later realized that the complete integrability of the model had a deep group theoretical explanation, that the simple pairwise interaction was the signature of an underlying structure: namely the root lattice of the Lie algebras $A_{N}$, and that all the relevant properties (complete integrability, mapping to a Laplace-Beltrami operator of a suitable symmetric space) still hold for potentials constructed by means of any root lattice canonically associated to a simple Lie algebra [19]. Let us see more precisely how this construction works.

Let us call $\mathcal{V}$ the $N$ dimensional space defined by the coordinates $\left\{x_{i}\right\}$ and $x=\left(x_{1}, \cdots x_{N}\right)$ a vector in $\mathcal{V}$. Let $R=\{\alpha\}$ be a root system in $\mathcal{V}$, and $R_{+}$the subsystem of positive roots of $R$. Let us denote with $x_{\alpha}$ the scalar product $(\alpha, x)$. Then the general form of the CS Hamiltonian is

$$
H=-\frac{1}{2} \sum_{i=1}^{N} \frac{\partial^{2}}{\partial^{2} x_{i}}+\sum_{\alpha \in R_{+}} \frac{g_{\alpha}^{2}}{\sinh ^{2}\left(x_{\alpha}\right)}
$$

where the couplings $g_{\alpha}^{2}$ are the same for equivalent roots, namely for those roots which are connected with each other by transformations of the Coxeter group $W$ of the root system. To clarify this rather abstract definition let us see two examples, obtained using the root lattices $A_{N}$ and $C_{N}$ (in the following $\left\{e_{i}, \cdots e_{N}\right\}$ denote a canonical basis in the space $\mathbf{R}^{n}$ ).

$A_{N}$ : This root system is obtained by taking a hyperplane in $\mathbf{R}^{N+1}$ for which $x_{1}+x_{2}+\cdots x_{N+1}=1$. Then the root system $R$ is given by: $R=\left\{e_{i}-e_{j}, i \neq\right.$ $j\}$. In this case $\mathrm{W}$ is the permutation group of the set $\left\{e_{i}\right\}$. The corresponding Hamiltonian is:

$$
H=-\frac{1}{2} \sum_{i=1}^{N} \frac{\partial^{2}}{\partial^{2} x_{i}}+\sum_{i<j} \frac{g^{2}}{\sinh ^{2}\left(x_{i}-x_{j}\right)}
$$

This is the model originally considered in [6]

$C_{N}$ : This root system is $R=\left\{ \pm 2 e_{i}, \pm e_{i} \pm e_{j}, i \neq j\right\}$, in this case $\mathrm{W}$ is the product of the permutation group and the group of transformations which change the sign of the vectors $\left\{e_{i}\right\}$. The corresponding Hamiltonian is: 


$$
\begin{array}{r}
H=-\frac{1}{2} \sum_{i=1}^{N} \frac{\partial^{2}}{\partial^{2} x_{i}}+\sum_{i} \frac{g_{2}^{2}}{\sinh ^{2}\left(2 x_{i}\right)} \\
+\sum_{i<j}\left(\frac{g_{1}^{2}}{\sinh ^{2}\left(x_{i}-x_{j}\right)}+\frac{g_{1}^{2}}{\sinh ^{2}\left(x_{i}+x_{j}\right)}\right)
\end{array}
$$

This is the model which we shall study in the following.

By using simple identities among hyperbolic functions eq.(30) can be rewritten as follows:

$$
\begin{aligned}
H & =-\frac{1}{2} \sum_{i=1}^{N} \frac{\partial^{2}}{\partial^{2} x_{i}}+\sum_{i} \frac{g_{2}^{2}}{\sinh ^{2}\left(2 x_{i}\right)}+c \\
& +2 g_{1}^{2} \sum_{i<j} \frac{\sinh ^{2}\left(2 x_{i}\right)+\sinh ^{2}\left(2 x_{j}\right)}{\left(\cosh \left(2 x_{i}\right)-\cosh \left(2 x_{j}\right)\right)^{2}}
\end{aligned}
$$

with $c$ an irrelevant constant. By setting

$$
g_{2}^{2}=-1 / 2, \quad g_{1}^{2}=\frac{\beta(\beta-2)}{4}
$$

we see that eq.(30) coincides (apart from the overall factor $1 / \gamma$ ) with $\mathcal{H}$ in eq.(26).

As we mentioned above, the relevant feature of the CS hamiltonian (30) is that it can be mapped into the radial part $B$ of a Laplace-Beltrami operator (see for instance Appendix D of ref. [19]) of a suitable symmetric space

$$
H=\xi(x)\left[\frac{1}{2}\left(B+\rho^{2}\right)\right] \xi(x)^{-1}
$$

with $B$ defined by eq.(21) and $\rho$ a constant term which we shall neglect in the following. The particular symmetric space is uniquely fixed by the root lattice underlying the CS Hamiltonian and by the coupling constant $g_{\alpha}$ In fact it is well known that all the irreducible symmetric spaces of classical type can be classified with essentially the same techniques used for the Lie algebras. They fall into 11 classes labelled by the type of root system and by the multiplicities of the various roots [12]. Some of these spaces (those relevant for our discussion) are listed in tab.2 and 3 with their root multiplicities.

These multiplicities are related to the coupling constants by 19

$$
g_{\alpha}^{2}=\frac{m_{\alpha}\left(m_{\alpha}-2\right)}{8}|\alpha|^{2}
$$

where $|\alpha|$ is the length of the root $\alpha$ and $m_{\alpha}$ its multiplicity. Only for these special values of $g_{\alpha}^{2}$ the mapping (33) is possible. For the three ensembles in which we are interested we have $m_{\alpha}=\beta$ for the short roots (those of the type $\left\{ \pm e_{i} \pm e_{j}\right\}$ ) and 


\begin{tabular}{|c|c|c|}
\hline$G$ & $H$ & $\beta$ \\
\hline$S L(N, \mathbf{R})$ & $S O(N)$ & 1 \\
$S L(N, \mathbf{C})$ & $S U(N)$ & 2 \\
$S U^{*}(N)$ & $U S p(2 N)$ & 4 \\
\hline
\end{tabular}

Table 2: Irreducible symmetric spaces of type $A_{N}$. In the first two columns the group $G$ and the maximal subgroup $H$ which define the symmetric space. All these spaces are labelled by the Dynkin diagram $A_{N-1}$. In the last column the multiplicity $\beta$ of the roots

\begin{tabular}{|c|c|c|c|}
\hline$G$ & $H$ & $\beta$ & $\eta$ \\
\hline$S p(2 N, \mathbf{R})$ & $U(N)$ & 1 & 1 \\
$S U(N, N)$ & $S U(N) \otimes S U(N) \otimes U(1)$ & 2 & 1 \\
$S O^{*}(4 N)$ & $U(2 N)$ & 4 & 1 \\
$U S p(2 N, 2 N)$ & $U S p(2 N) \otimes U S p(2 N)$ & 4 & 3 \\
$S p(2 N, \mathbf{C})$ & $U S p(2 N)$ & 2 & 2 \\
\hline
\end{tabular}

Table 3: Irreducible symmetric spaces of type $C_{N}$. In the first two columns the group $G$ and the maximal subgroup $H$ which define the symmetric space. All these spaces are labelled by the Dynkin diagram $C_{N}$. In the third column the multiplicity $\beta$ of the ordinary roots of $C_{N}$. In the last column the multiplicity $\eta$ of the long root.

$m_{\alpha}=1$ for the long roots (those of the type $\left\{2 e_{i}\right\}$ ), which if inserted in eq.(34) exactly give the values of eq.(32). In this way we see that the index $\beta$ has a deep group theoretical meaning, since it denotes the multiplicity of the (ordinary) roots of the symmetric space in which the transfer matrix diffuses as $L$ increases. The mapping described by eq. (33) is exactly the one found in ref. [18].

All the symmetric spaces listed in tab.2 and 3 are spaces of negative curvature. For each one of them there is a counterpart of positive curvature, with all other properties (in particular the root lattice structure) unchanged. For instance we have:

$$
\frac{S L(N, \mathbf{R})}{S O(N)} \rightarrow \frac{S U(N)}{S O(N)}
$$

In the context of CS models one moves from negative to positive curvature symmetric spaces by changing the sinh-type interaction into the sin-type one. In the framework of RMT for disordered wires one has the same change moving from the TME to the S-matrix ensembles. In fact, as we mentioned above, the flux conservation constraint implies that the $S$ matrix belongs to a compact group. At this point, depending on the problem in which one is intersted, and consequently, on the parametrization which one choses for the (possibly generalized) eigenvalues one 
can find WDE's, which correspond to sin CS models of $A_{N}$ type, or the S-matrix ensembles described in [5] which correspond to $\sin$ CS model of $C_{N}$ type.

\section{5 solution of the DMPK equation}

The most important application of the above described equivalence between TME and CS models is that, by using some recent results on the CS models one can obtain several important properties of the DMPK equation: solve it exactly in the case $\beta=$ 2 and find approximate asymptotic solutions for $\beta=1,4$. This result was obtained in 116 and we shall describe here the main steps of that solution. According to eq. 23. if $\Phi_{k}(x), x=\left\{x_{1}, \cdots, x_{N}\right\}, k=\left\{k_{1}, \cdots, k_{N}\right\}$ is an eigenfunction of $B$ with eigenvalue $k^{2}$, then $\xi(x)^{2} \Phi_{k}(x)$ will be an eigenfunction of the DMPK operator with eigenvalue $k^{2} /(2 \gamma)$. These eigenfunctions of the $B$ operator are known in the literature as "zonal spherical functions". In the following we shall use three important properties of these functions (see [20]).

1] By means of the zonal spherical functions one can define the analog of the Fourier transform on symmetric spaces:

$$
f(x)=\int \bar{f}(k) \Phi_{k}(x) \frac{d k}{|c(k)|^{2}}
$$

(where we have neglected an irrelevant multiplicative constant) and in the three cases which are of interest for us:

$$
|c(k)|^{2}=|\Delta(k)|^{2} \prod_{j}\left|\frac{\Gamma\left(i \frac{k_{j}}{2}\right)}{\Gamma\left(\frac{1}{2}+i \frac{k_{j}}{2}\right)}\right|^{2}
$$

with

$$
|\Delta(k)|^{2}=\prod_{m<j}\left|\frac{\Gamma\left(i \frac{k_{m}-k_{j}}{2}\right) \Gamma\left(i \frac{k_{m}+k_{j}}{2}\right)}{\Gamma\left(\frac{\beta}{2}+i \frac{k_{m}-k_{j}}{2}\right) \Gamma\left(\frac{\beta}{2}+i \frac{k_{m}+k_{j}}{2}\right)}\right|^{2}
$$

where $\Gamma$ denotes the Euler gamma function.

2] for large values of $x, \Phi_{k}(x)$ has the following asymptotic behaviour:

$$
\Phi_{k}(x) \sim \frac{1}{\xi(x)}\left(\sum_{r \in W} c(r k) e^{i(r k, x)}\right),
$$

where $r k$ is the vector obtained acting with $r \in W$ on $k$. The important feature of eq. (39) is that it is valid for all values of $k$.

$3]$ in the case $\beta=2$ the explicit form of $\Phi_{k}(x)$ is known [19, 21]:

$$
\Phi_{k}(x)=\frac{\operatorname{det}\left[Q_{m}^{j}\right]}{\prod_{i<j}\left[\left(k_{i}^{2}-k_{j}^{2}\right)\left(\sinh ^{2} x_{i}-\sinh ^{2} x_{j}\right)\right]}
$$


where the matrix elements of $Q$ are:

$$
Q_{m}^{j}=\mathrm{F}\left(\frac{1}{2}\left(1+i k_{m}\right), \frac{1}{2}\left(1-i k_{m}\right), 1 ;-\sinh ^{2} x_{j}\right)
$$

and $F(a, b, c ; z)$ is the hypergeometric function.

Eq.s(23,36-38) allow to write the $s$-evolution of $P\left(\left\{x_{n}\right\}, s\right)$ from given initial conditions (described by the function $\left.\bar{f}_{0}(k)\right)$ as follows:

$$
P\left(\left\{x_{n}\right\}, s\right)=[\xi(x)]^{2} \int \bar{f}_{0}(k) e^{-\frac{k^{2}}{2 \gamma} s} \Phi_{k}(x) \frac{d k}{|c(k)|^{2}} .
$$

By inserting the explicit expression of $|c(k)|^{2}$ and by using the identity:

$$
\left|\frac{\Gamma\left(\frac{1}{2}+i \frac{k}{2}\right)}{\Gamma\left(i \frac{k}{2}\right)}\right|^{2}=\frac{k}{2} \tanh \frac{\pi k}{2}
$$

we end up with the following general expression for $P\left(\left\{x_{n}\right\}, s\right)$ with ballistic initial conditions (which, due to the normalization of $\Phi_{k}(x)$, simply amount to choosing $\bar{f}_{0}(k)=$ const $)$ :

$$
\begin{aligned}
& P\left(\left\{x_{n}\right\}, s\right)=[\xi(x)]^{2} \int d k e^{-\frac{k^{2}}{2 \gamma} s} \frac{\Phi_{k}(x)}{|\Delta(k)|^{2}} \\
& \prod_{j} k_{j} \tanh \left(\frac{\pi k_{j}}{2}\right)
\end{aligned}
$$

This expression is rather abstract, but it can be made more explicit by using the properties [2] and [3] listed above. In the $\beta=2$ case we can insert the explicit expression for $\Phi_{k}(x)$, given in eq.s(40,41), into eq.(44). By using the identity

$$
\mathrm{P}_{\nu}(z)=F(-\nu, \nu+1,1 ;(1-z) / 2)
$$

we exactly obtain (as expected) the solution, found by Beenakker and Rejaei in the same case [18].

In the other two cases $\beta=1,4$, if $x$ is large (and in our framework this means $x^{2}>(2 s) / \gamma$ ) we may insert the asymptotic expansion (39) into eq.(44). The resulting behaviour of $P\left(\left\{x_{n}\right\}, s\right)$ will depend on the chosen (metallic or insulating) regime for $k$. Let us look at the two cases separately.

Insulating regime $(k \ll 1)$.

In the $k \rightarrow 0$ limit the $\Gamma$ functions in eq.(39) can be approximated according to:

$$
\frac{\Gamma\left(\frac{\beta}{2}+i y\right)}{\Gamma(i y)} \sim_{y \rightarrow 0} i y \quad, \quad \beta \in\{1,2,4\} .
$$


Then, by rewriting both the product $\prod_{i<j}\left(k_{i}^{2}-k_{j}^{2}\right)$, and the sum over the exponentials in (39) as determinants, the integration over $k$ becomes straightforward and gives:

$$
\begin{aligned}
P\left(\left\{x_{n}\right\}, s\right) & =\prod_{i<j}\left|\sinh ^{2} x_{j}-\sinh ^{2} x_{i}\right|^{\frac{\beta}{2}}\left[\left(x_{j}^{2}-x_{i}^{2}\right)\right] \\
& \times \prod_{i}\left[\exp \left(-x_{i}^{2} \gamma /(2 s)\right) x_{i}\left(\sinh 2 x_{i}\right)^{1 / 2}\right] .
\end{aligned}
$$

Ordering the $x_{n}$ 's from small to large and using the fact that in this regime $1 \ll x_{1} \ll x_{2} \ll \cdots \ll x_{N}$ we can approximate the eigenvalue distribution as follows:

$$
P\left(\left\{x_{n}\right\}, s\right)=\prod_{i=1}^{N} \exp \left[-(\gamma /(2 s))\left(x_{i}-\bar{x}_{i}\right)^{2}\right] .
$$

where $\bar{x}_{n}=\frac{s}{\gamma}(1+\beta(n-1))$, in agreement with the result obtained by Pichard, 22 by directly solving the DMPK equation in this regime.

Metallic regime $(k \gg 1)$.

In this case one must use the asymptotic expansion:

$$
\frac{\Gamma\left(\frac{\beta}{2}+i y\right)}{\Gamma(i y)} \sim_{y \rightarrow \infty}|y|^{\frac{\beta}{2}} e^{\frac{i \pi \beta}{4}}, \quad \beta \in\{1,2,4\}
$$

The integration over $k$ is less simple in this case and, (to be consistent with the regime of validity of eq. (39)) in the resulting expression only the highest powers of $\left(x \sqrt{\frac{\gamma}{2 s}}\right)$ must be taken into account. We find:

$$
\begin{aligned}
P\left(\left\{x_{n}\right\}, s\right) & =\prod_{i<j}\left|\sinh ^{2} x_{j}-\sinh ^{2} x_{i}\right|^{\frac{\beta}{2}}\left|x_{j}^{2}-x_{i}^{2}\right|^{\frac{\beta}{2}} \\
\times & \prod_{i}\left[\exp \left(-x_{i}^{2} \gamma /(2 s)\right)\left(x_{i} \sinh 2 x_{i}\right)^{1 / 2}\right] .
\end{aligned}
$$

In agreement with the exact result of [18 for $\beta=2$ and with the $\beta$ dependence found by Chalker and Macêdo 23] through a direct integration of the DMPK equation.

According to ref. [19] and ref. [20], the regime of validity of eq.s(47) and (50) is $x^{2}>(2 s) / \gamma$, which gives in in the large $N$ limit $x^{2}>(2 s) /(\beta N)$. Notice however that eq.(39) is only the first term of a series which converges absolutely to $\Phi_{k}(x)$ for all values of $k$ (see sect. 8 of ref. [19] and ref. [20]). The coefficients of this series can be constructed recursively, thus allowing to study the behaviour of eq. (44) even for values of $x$ smaller than the above mentioned threshold. 


\section{Conclusions and Perspectives}

We have shown that there is a deep connection between CS models and the RMT approach to disordered wires. In particular we have shown that sinh CS models of $C_{N}$ type correspond to TME's, that sin CS models of $C_{N}$ type correspond to the S-matrix ensembles in the parametrization of ref [5] and that ordinary WDE's correspond to sin CS models of $A_{N}$ type. Several properties of the TME's can be obtained by exploiting this connection. In particular exact or approximate solution of the DMPK equation can be obtained.

Another interesting application of this correspondence can be found in the insulating regime. Looking at tab.2 and tab.3 we see that the most relevant feature of the $C_{N}$ symmetric spaces with respect of the $A_{N}$ ones is that they are described by two critical indices instead of one. This is due to the fact that the $C_{N}$ Dynkin diagrams have two types of roots, each with its particular multiplicity. We shall call this second index $\eta$ in the following. This peculiar feature of these ensembles is usually ignored because in the weakly localized regime it is only the value of $\beta$ which matters, and also because the three cases which have been studied up to now (the first three lines of tab.1) have the same value of the second index $\eta=1$, thus leading to the same DMPK equation (with no explicit $\eta$ dependence).

However in the insulating regime also the index $\eta$ becomes important and can be directly measured by looking for instance at the ratio [24]

$$
\frac{\operatorname{var}(\log g)}{<\log g>}=\frac{2}{\eta}
$$

In fact, while in the weakly localized regime all the relevant physical properties

are completely determined by the level statistics (namely the value of $\beta$ ), when extending the RMT approach to the insulating regime, due to the fact that in this case the conductance is dominated by the lowest eigenvalue all the details of the chosen RMT model (number of degrees of freedom, possibly the presence of new critical indices) become important [24]. This observation could open a new interesting field of application of the Calogero-Sutherland techniques in the physics of disordered wires

Acknowledgements I want to warmly thank G. Zemba and F. Gliozzi for their constant help and encouragement.

\section{References}

[1] For reviews see Mesoscopic Phenomena in Solids (North-Holland, Amsterdam, 1991) ed. by B. L. Altshuler, P. A. Lee, and R. A. Webb.

[2] Y. Imry, Europhys. Lett. 1, 249 (1986). 
[3] K. A. Muttalib, J.-L. Pichard, and A. D. Stone, Phys. Rev. Lett. 59, 2475 (1987).

[4] F.J. Dyson, J. Math. Phys. 3 (1962) 1191.

[5] K. Frahm and J.L. Pichard, preprint cond-mat/9504097

[6] F. Calogero, J. Math. Phys. 10, 2191 (1969); B. Sutherland, J. Math. Phys. 12, 246 (1971); 12, 251 (1971).

[7] R. Landauer, Phil. Mag. 21, (1970) 863 .

[8] D.S.Fisher and P.A.Lee, Phys. Rev. B 23 (1981) 6851.

[9] K.B. Efetov and A.I. Larkin, Sov. Phys. JETP 58, 444 (1983); K.B. Efetov, Adv. Phys. 32, 53 (1983).

[10] P. A. Mello, P. Pereyra, and N. Kumar, Ann. Phys. 181, 290 (1988).

[11] J. T. Chalker and A. M. S. Macêdo, Phys. Rev. B 46, 14985 (1992).

[12] S. Helgason, Differential Geometry, Lie Groups and Symmetric Spaces (Academic, 1978)

[13] O. N. Dorokhov, Pis'ma Zh. Eksp. Teor. Fiz. 36, 259 (1982) [JETP Lett. 36, $318(1982)]$.

[14] P. A. Mello and A. D. Stone, Phys. Rev. B 44, 3559 (1991).

[15] A. Hüffmann, J. Phys. A 23, 5733 (1990).

[16] M. Caselle, Phys. Rev. Lett. 742776 (1995).

[17] A. Comtet and C. Monthus, preprint cond-mat/9506024

[18] C. W. J. Beenakker and B. Rejaei, Phys. Rev. Lett. 71, 3689 (1993); Phys. Rev. B 49, 7499 (1994).

[19] M. A. Olshanetsky and A. M. Perelomov, Phys. Rep. 94, 313 (1984).

[20] Harish-Chandra, Amer. J. Math 80241 (1958); 80553 (1958).

[21] F. A. Berezin and F. I. Karpelevick, Dokl. Akad. Nauk SSSR 118, 9 (1958).

[22] J.-L. Pichard, in Quantum Coherence in Mesoscopic Systems, edited by B. Kramer, NATO ASI Series B254 (Plenum, New York, 1991).

[23] J. T. Chalker and A. M. S. Macêdo, Phys. Rev. Lett. 71, 3693 (1993).

[24] M. Caselle, preprint cond-mat/9505068 